\title{
Assembly Pathway of Desmoglein 3 to Desmosomes and Its Perturbation by Pemphigus Vulgaris-IgG in Cultured Keratinocytes, as Revealed by Time-Lapsed Labeling Immunoelectron Microscopy
}

\author{
Miki Sato, Yumi Aoyama, and Yasuo Kitajima \\ Department of Dermatology, Gifu University School of Medicine, Gifu, Japan
}

SUMMARY: To determine the assembly pathway of desmoglein 3 (Dsg3) into desmosomes and the subsequent effects of pemphigus vulgaris immunoglobulin G (PV-lgG) on such, we employed a time-lapsed labeling for FITC/Rhodamine (Rod) double-stained immunofluorescence and 5-nm/10-nm gold double-stained immunoelectron microscopy by using PV-lgG, which was confirmed to react specifically Dsg3. Cells from a human squamous cell carcinoma cell line (DJM-1) were first treated briefly with PV-IgG (3 min), then incubated in either anti-human IgG-FITC or 5-nm gold antibody-containing medium (5 min), followed by a 60-minute chase in normal medium without antibodies. The same cells were reincubated with PV-lgG medium for 3 minutes, followed by either anti-human IgG-Rod or 10-nm gold antibodies for 5 minutes. Using this method, FITC and 5-nm gold particles show the fate of Dsg3-PV-lgG complexes during the following 60-minute chase. IgG-Rod or 10-nm gold particles, which are bound during the last 5 minutes of the chase, show Dsg3 molecules newly expressed on the cell surface during the 60-minute-chase period. Initially, Dsg3 formed two types of small clusters on the nondesmosomal plasma membrane, ie, either half-desmosome-like clusters with keratin intermediate filament (KIF) attachment or simple clusters without KIF attachment. The PV-IgG binding to Dsg3 caused the internalization of the simple clusters into endosomes, but not the half-desmosome-like clusters. After the 60-minute-chase period, both types of cell surface Dsg3 clusters were labeled with only 10-nm gold, suggesting that new Dsg3 molecules were being delivered to the cell surface. Desmosomes were labeled with both 5-nm gold and 10-nm gold, whereas the half-desmosome-like clusters were labeled with only 10-nm gold, suggesting that the desmosomes themselves were not split. These results suggest that Dsg3 first forms simple clusters, followed by KIF-attachment, and then becomes integrated into desmosomes, and that PV-lgG-induced internalization of the nondesmosomal simple clusters of Dsg3 may represent the primary effects of PV-IgG on keratinocytes. (Lab Invest 2000, 80:1583-1592).

$D$ esmosomes are intercellular adhering junctions that provide the plasma membrane with anchorage sites consisting of clusters of desmosomal cadherins and several plaque proteins, for the attachment of keratin intermediate-sized filaments (KIF) (Buxton et al, 1993; Koch and Franke, 1994; Legan et al, 1992). These desmosomal cadherins consist of two major transmembrane glycoproteins, ie, the desmogleins 1 to 3 (Dsg 1, 2, and 3) and the desmocollins 1 to 3 (Dsc 1,2 , and 3). The intracytoplasmic domains of these proteins serve as binding sites for plakoglobin, desmoplakins, and other desmosome-specific plaque proteins (Amagai, 1995; Koch and Franke, 1994). Dsg 1 (160 kDa) and Dsg $3(130 \mathrm{kd})$ are now known as the target molecules for pemphigus foliaceus (PF) and pemphigus vulgaris (PV), respectively (Amagai, 1995; Stanley, 1992). Dsg 1 is expressed mainly in the upper epidermis, whereas Dsg 3 is primarily in the lower-tomiddle epidermis (Amagai et al, 1996; Arnemann et al,

Received July 25, 2000.

Address reprint requests to: Dr. Yasuo Kitajima, Department of Dermatology, Gifu University School of Medicine, 40 Tsukasamachi, Gifu City, Gifu 500-8705, Japan. FAX: 8158265 9017; E-mail: yaskitaj@gumail.cc.gifu-u.ac.jp
1993; Shimizu et al, 1995). The intracytoplasmic plaques consist of several groups of proteins that may roughly divided into three groups: (a) the plakins (Ruhrberg and Watt, 1997), ie, desmoplakin 1 and 2 (Mueller and Franke, 1983), plectin (Wiche et al, 1991), periplakin (Ruhrberg et al, 1997), and envoplakin (Ruhrberg et al, 1996); (b) proteins homologous to catenins, ie, plakoglobin (Cowin et al, 1986) and plakophilin (Heid et al, 1994); and (c) others proteins such as desmoyokin (Hieda et al, 1989), desmocalmin (Tsukita and Tsukita, 1985), and pinin (Ouyang and Sugrue, 1996). Most of these molecules appear to play some direct or indirect roles as linkers between the KIFs and the transmembrane core proteins or desmosomal cadherins. Although the molecular structures of desmosomes have been rather well understood, as mentioned above, it is still unclear how these desmosomal molecules assemble into mature desmosomes.

Pemphigus is a group of an autoimmune blistering diseases, two major types of which are PV and PF. PV is caused by autoantibodies against Dsg 3 and is characterized by flaccid blisters and erosions caused by acantholysis in the deeper (mainly suprabasal) epidermis. Conversely, PF is caused by autoantibodies against Dsg1 and is characterized by crusted and 
scaly lesions caused by acantholysis in the superficial epidermis (Amagai, 1995; Stanley, 1992). Although recent molecular genetic studies revealed the target molecules of pathogenic autoantibodies, the mechanisms leading to acantholysis remain unresolved. Two possible mechanisms for blistering have been proposed, ie, direct impairment of adhesive functions and desmogleins (Koch et al, 1997), or cell biological responses to antibody-binding to desmogleins, leading to acantholysis (Kitajima et al, 1987; Kitajima, 1996).

In this paper, we address the following four questions: (a) whether or not Dsg3 is distributed on the nondesmosomal (interdesmosomal) plasma membrane with or without binding to KIF in cultured keratinocytes; (b) if so, how these Dsg3 molecules are then assembled into desmosomes; (c) what effects on this process are exerted by pemphigus vulgaris immunoglobulin G (PV-lgG) binding to these Dsg3 molecules; and, finally, (d) whether direct binding of PV-IgG to Dsg3 in desmosomes splits the desmosome. To track the dynamic distribution and ultimate fate of Dsg3, we employed a time-lapsed labeling method, both for FITC/Rhodamine (Rod) double-stained immunofluorescence and for 5-nm/10-nm gold doublestained immunoelectron microscopy, by using PV-IgG followed by FITC, Rod, 5-nm gold- or 10-nm goldlabeled secondary antibodies against human IgG. The time-lapsed labeling studies showed that Dsg3 initially forms simple small clusters, without KIF-attachment, on the external aspect of nondesmosomal plasma membrane. This clustering is followed by KIFattachment on the intracytoplasmic surface, leading to the formation of half-desmosome-like clusters before integration of Dsg3 into desmosomes, as previously suggested in low $\mathrm{Ca}^{++}$-grown cells from a human keratinocyte line (HaCaT) (Demlehner et al, 1995). Moreover, the PV-IgG binding to Dsg3 lead to endosomal internalization of these simple Dsg3 clusters, without KIF-attachment, but not of the halfdesmosome-like clusters in which KIF-attachment already had occurred. $\mathrm{PV}$-IgG-induced internalization of Dsg3 plays a significant role in the formation of Dsg3-depleted desmosomes following PV-IgG stimulation of cultured cells from a human squamous cell carcinoma cell line (DJM-1)(Aoyama and Kitajima, 1999).

\section{Results}

\section{PV-IgG Causes Rapid Aggregation and Internalization of Nondesmosomal Dsg3 as Observed by Immunofluorescence Microscopy}

To determine the temporal changes in Dsg3 localization on the cell surface after binding of PV-lgG to Dsg3, we performed a chase experiment of Dsg3 in DJM-1 cells. When cells were briefly incubated with medium containing PV-lgG (3 min) and labeled with fresh medium containing antihuman IgG-FITC for 5 minutes, FITC fluorescence was distributed as a linear line between cells and homogeneously, although very faintly, over the entire cell surface, including the non- adherent cell surface (Fig. 1a). However, when cells that had been pulse-treated ( $3 \mathrm{~min}$ ) with PV-lgG and pulse-stained (5 min) with antihuman IgG-FITC were further incubated for 60 minutes in PV-lgG-free medium, a distinct fluorescent punctuate pattern was generated, not only along the cell-cell contacts, but also on the nonadherent cell surface (Fig. 1b). As an alternative experiment, when pulse-PV-lgG-treated cells were chased for 60 minutes and stained with antihuman IgG-FITC, after being fixed (3.0\% paraformaldehyde in $0.1 \mathrm{M}$ sodium phosphate buffer) and permeabilized $(0.05 \%$ Triton $\mathrm{X}-100)$, the same punctuate pattern of FITC was observed. This generation of a punctuate pattern of FITC implicates the formation of Dsg3 aggregates on the free cell surface, suggesting that Dsg3 is freely distributed also on free (nondesmosomal) cell surfaces.

To determine whether these spots on the free cell surface of non-cell-cell contacts are actually located on the cell surface or internalized into cells as endosomes, cells were first pulse-treated with PV-lgG (3 $\min$ ) and stained with antihuman IgG-FITC in culture medium (5 min) and incubated for 60 minutes in antibody-free culture medium. Then, these cells were briefly reincubated with PV-IgG (5 min), after the 60-minute chase in antibody-free culture medium, and stained with fresh medium containing antihuman IgGRhodamine (Rod) goat antibodies (5 min) before fixation. This time-lapsed double-staining showed that FITC-positive spots visible along the cell-cell contacts, which are thought to correspond to desmosomes, also were stained with red Rod fluorescence. Conversely, many of the FITC-positive sites in the central domains of cells were not co-stained with red Rod (Fig. 1, c and d). This suggests that the FITC single-labeled spots represent already-internalized Dsg3, because the newly incubated PV-lgG and antihuman IgG-Rod cannot penetrate into the cell in living cells in culture medium. Negative controls that were carried out at the same time using normal human IgG showed no staining with FITC and Rod (data not shown).

\section{Distribution of Dsg3 on the Nondesmosomal Plasma Membrane as Observed by Immunoelectron Microscopy: Simple Clusters and Half-Desmosome-Like Clusters}

To determine the natural intact localization of Dsg3 on the cell surface, we first briefly fixed the cells with $3 \%$ paraformaldehyde in $0.1 \mathrm{M}$ sodium phosphate buffer and stained Dsg3 using PV-IgG (ie, anti-Dsg3) and antihuman IgG labeled with $10 \mathrm{~nm}$-gold particles.

On the cell surface of DJM-1 cells fixed with $3 \%$ paraformaldehyde in $0.1 \mathrm{~m}$ sodium phosphate buffer, Dsg3 was distributed both as simple small clusters without evidence of KIF-attachment to the cytoplasmic face of the clusters (Fig. 2a) and as small halfdesmosome-like clusters with KIF-attachment and intracytoplasmic dense plate (attachment plaque-like structure)(Fig. 2b). The average number of $10-\mathrm{nm}$ gold particles ranges from 10 to 30 per plaque structure. Single-gold particle deposits were rarely detected, 

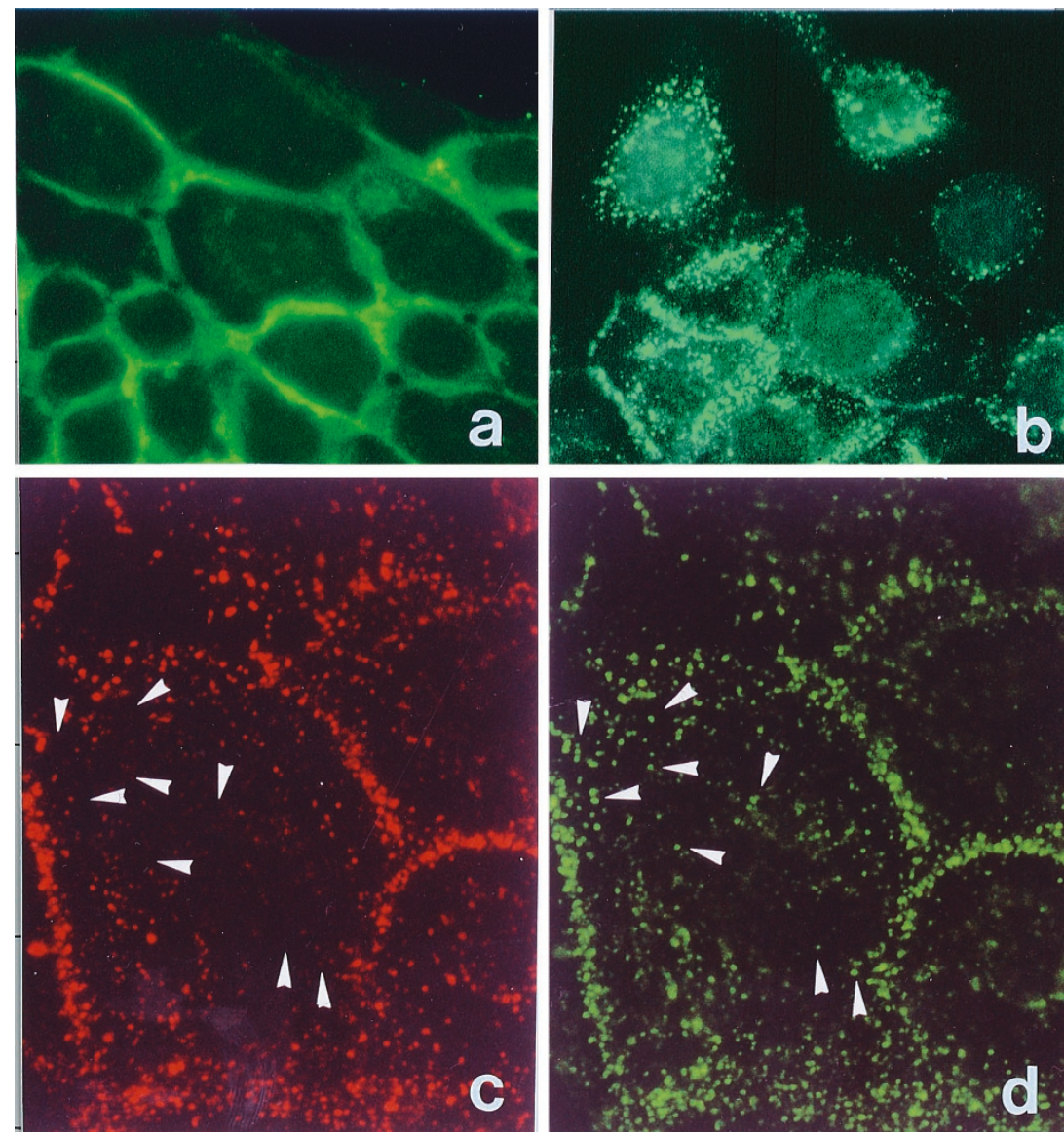

\section{Figure 1.}

Immunofluorescence micrographs showing pemphigus vulgaris immunoglobulin G (PV-lgG)-induced lateral aggregation (Panels a and $b$ ) and internalization (Pane/s $c$ and $d$ ) of desmoglein 3 (Dsg3) on the cell surface of cultured DJM-1 cells. Cells were stimulated with PV-lgG-containing medium for a short pulse time of 3 minutes, followed by washing with PBS, and stained with antihuman IgG-FITC in culture medium for 5 minutes (Panel a). FITC is distributed as a linear line on the entire cell surface of cell-cell contact areas and diffusely, although very faintly, on the non-cell-cell contact cell surfaces (Panel a). However, when cells were further incubated in antibody-free medium for an additional 60 minutes after a pulse treatment with PV-IgG (3 min) and staining with antihuman IgG-FITC in culture medium (5 min), a distinct punctuated-label pattern was evident on the entire cell surface, including non-cell-cell contact (free cell surface) areas (Panel $b$ ). To determine whether these spots on the free cell surface of non-cell-cell contacts were actually located on the cell surface or internalized within the cells as endosomes, cells were briefly reincubated with PV-IgG $(5 \mathrm{~min})$ after the 60-minute chase in antibody-free culture medium and stained with a fresh medium containing antihuman IgG-Rod antibodies for 5 minutes. This time-lapsed double-staining showed that FITC-positive spots visible along the cell-cell contacts, which correspond to desmosomes, also were stained with red Rod fluorescence. Conversely, many of the FITC-positive sites in the central domains of cells were not co-stained with red Rod (Panels $c$ and $d$ ) suggesting that FITC-positive, Rod-negative dots were internalized as endosomes, to which the second labeling of PV-lgG could not penetrate.

suggesting that Dsg3 exists in clusters on the external cell surface of keratinocytes in culture. To confirm that these clusters of Dsg3 were not parts of the margins of desmosomes, a serial section consisting of 5 to 6 sections was made. The former clusters were seen over 2 to 3 thin serial sections (100 nm thick) without any of KIF on the cytoplasmic face of the plasma membrane, suggesting that these are not parts of desmosomes (Fig. 2, d to f). The latter clusters were also seen over 2 to 3 thin serial sections with evidence of KIF attachment, indicating also that they are not parts of desmosomes. Negative controls that were carried out at the same time using normal human IgG showed no deposits of gold particles (data not shown).

\section{PV-IgG Causes Internalization of Simple Dsg3 Clusters without KIF-Attachment as Endosomes}

When cells were pulse-stimulated with PV-IgG for 3 minutes, labeled with 5-nm gold, and chased in antibody-free fresh culture medium for 15 minutes, small clusters of gold particles were observed on the inner surface of the endosomal membranes without KIF-attachment (Fig. 3a). The inner content of these endosomes appeared clear and lacked gold labeling (Fig. 3a). A thorough examination revealed that no half-desmosome-like clusters of Dsg3 labeled with gold were found, suggesting that only the simple clusters of Dsg3 may be internalized. After a longer chase period of 60 minutes, the 5-nm gold particles in the endosomes were released from the inner surface of the endosome membrane and distributed randomly in the endosomes, whereas the inside of the endosomes became filled with a denser unknown electron substance(s) (Fig. 3b). Finally, at this time, gold particles were condensed into a few aggregates in the center of endosomes (Fig. 3c).

To further investigate the fate of Dsg3 after the binding of PV-lgG to Dsg3, we performed time-lapsed 


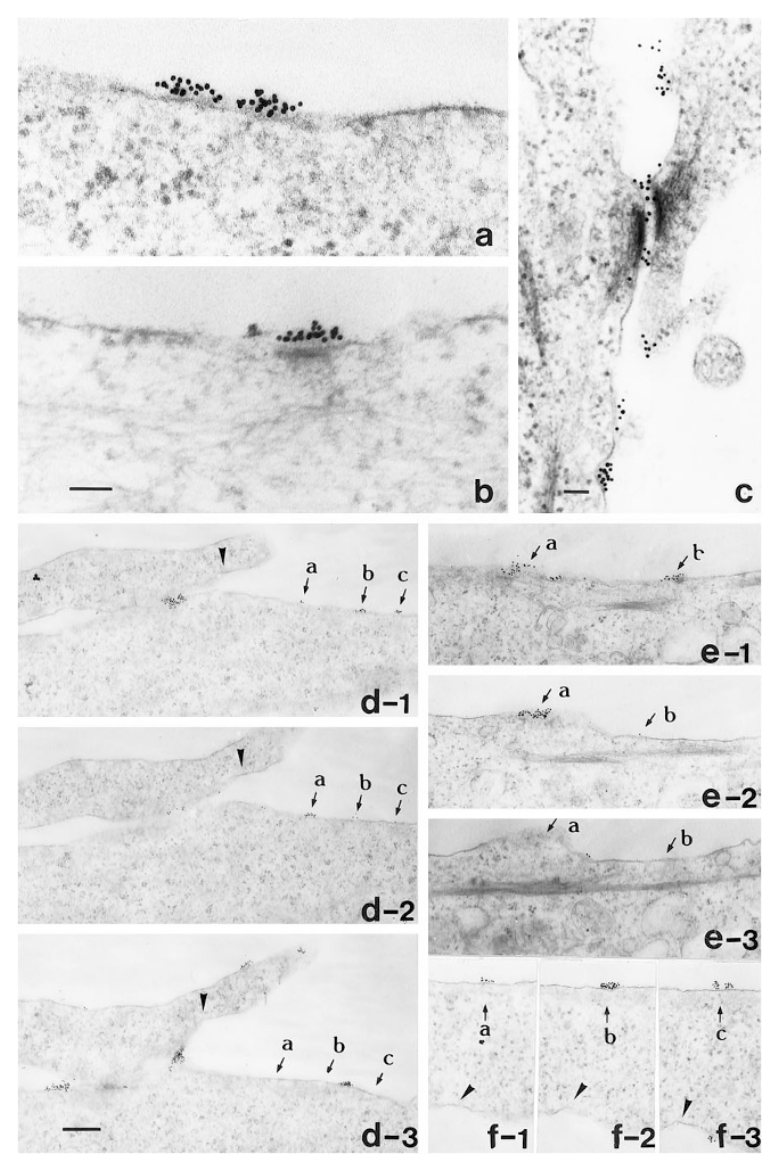

Figure 2.

Immunoelectron micrographs showing distribution of Dsg3 on the cell surface of DJM-1 cells. Prefixed cells were treated with PV-IgG and stained with antihuman IgG labeled with 10-nm gold. Anti-lgG gold particles bound to nondesmosomal plasma membrane to reveal two types of clusters, ie, simple clusters of gold particles without KIF attachment (Panel a) and halfdesmosome-like clusters with KIF-attachment and intracytoplasmic attachment plaque $(P a n e l b)$. Desmosomes were also labeled with PV-IgG and gold particles (Pane/ c). Three serial sections (Panels $d-1, d-2, d-3, f-1, f-2, f-3$ ) illustrate that the simple cluster of gold particles were not associated with KIF and were not a part of desmosomes and that half-desmosome-like clusters with KIF-attachment were not a part of desmosomes (Panels e-1, e-2, e-3). Scale bars $=0.1 \mu \mathrm{m}$ (Panels a to $c$ ) and $0.5 \mu \mathrm{m}$ (Panels $d$ and $f$ ).

double-staining of Dsg3 at the ultrastructural level. When cells are pulse-stimulated with PV-lgG for 3 minutes and labeled with 5-nm gold, followed by a chase in antibody-free medium for 60 minutes, and then relabeled with PV-lgG and antihuman IgG-10-nm gold particles, $10-\mathrm{nm}$ gold particles that lack concurrent 5-nm gold represent newly expressed Dsg3 clusters on the cell surface. This suggests that these 10-nm-gold Dsg3 clusters have arisen during the 60-minute chasing period. The 5-nm gold label contrives to represent older clusters of Dsg3 molecules formed before the chase period. At this time most of the gold particle clusters on the nondesmosomal cell surface were composed of 10-nm gold, whether or not they were attached with KIF on the inner aspects of their plasma membrane (Fig. 4). However, a few of these clusters contained both of 5-nm gold and 10-nm gold particles.

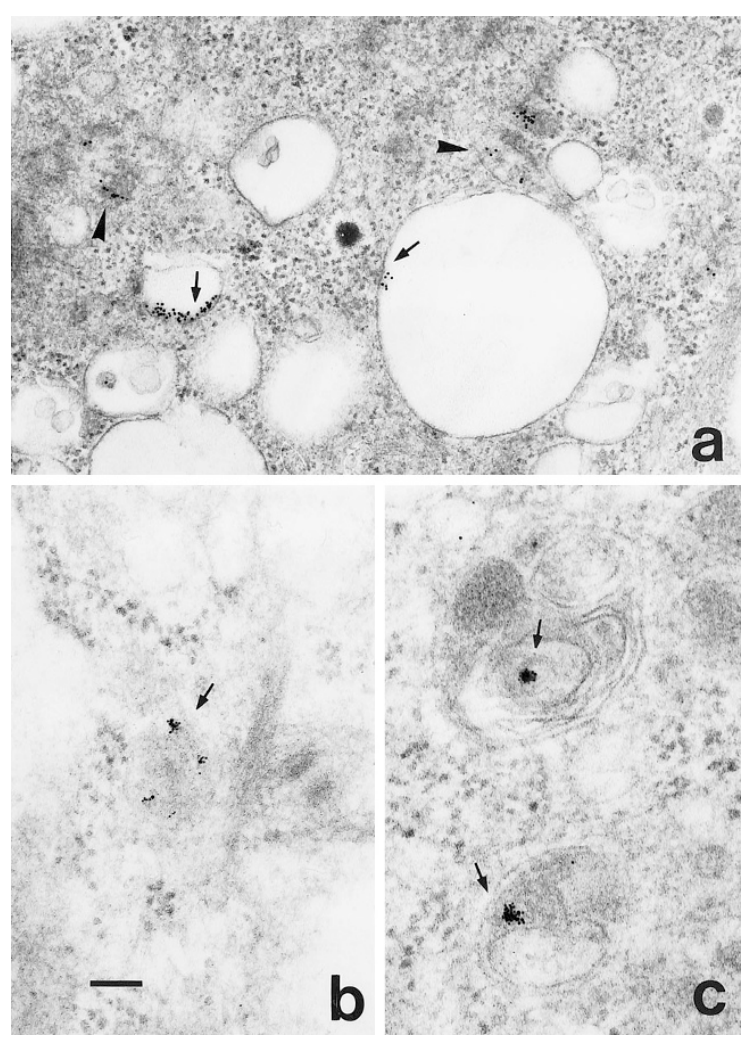

Figure 3.

Immunoelectron micrographs of DJM-1 cells treated with a short pulse ( $3 \mathrm{~min}$ ) of PV-IgG, stained with antihuman IgG-5-nm gold (5 min) and chased for 15 minutes or 60 minutes. Gold particles appear as attached to the inner surface of endosomes, which also reveal minimal content inside after a 15-minute chase (Panel a). However, after a 60-minute chase, gold particles were released from the inner surface of the endosomes (Panel $b$ ) and formed small aggregates within the endosomes (Panel $c$ ), the insides of which contained a more-electron-dense substance or membranous structures. Scale bar $=0.5$ $\mu \mathrm{m}$.

All desmosomes, as long as observed, were labeled with both $5-\mathrm{nm}$ gold and 10-nm gold particles, indicating that desmosomes existed continuously and were not newly formed during the chase of 60 minutes (Fig. 5). The fact that nearly all of half-desmosome-like clusters of Dsg3 were labeled with only 10-nm gold particles suggests that these half-desmosome-like clusters are not split desmosomes, ie, halfdesmosomes, but rather were formed during the 60minute chase period. Negative controls that were carried out at the same time using normal human IgG showed no deposits of gold particles (not shown).

\section{Discussion}

Although recent molecular studies have proved that the pathogenic antigen of PV-lgG is a transmembrane core protein of desmosomes, Dsg3 (Amagai, 1995; Stanley 1992), it remains unresolved whether Dsg3 is localized to the interdesmosomal (ie, nondesmosomal) cell membrane as well as to the desmosomes. Earlier studies by immunoperoxidase staining (Bedane C et al, 1996; Patel et al, 1984; Takahashi et al, 1985; Wolff and Schreiner, 1971), by ferritin label staining (Takigawa et al, 1978), or by post-embedding immu- 


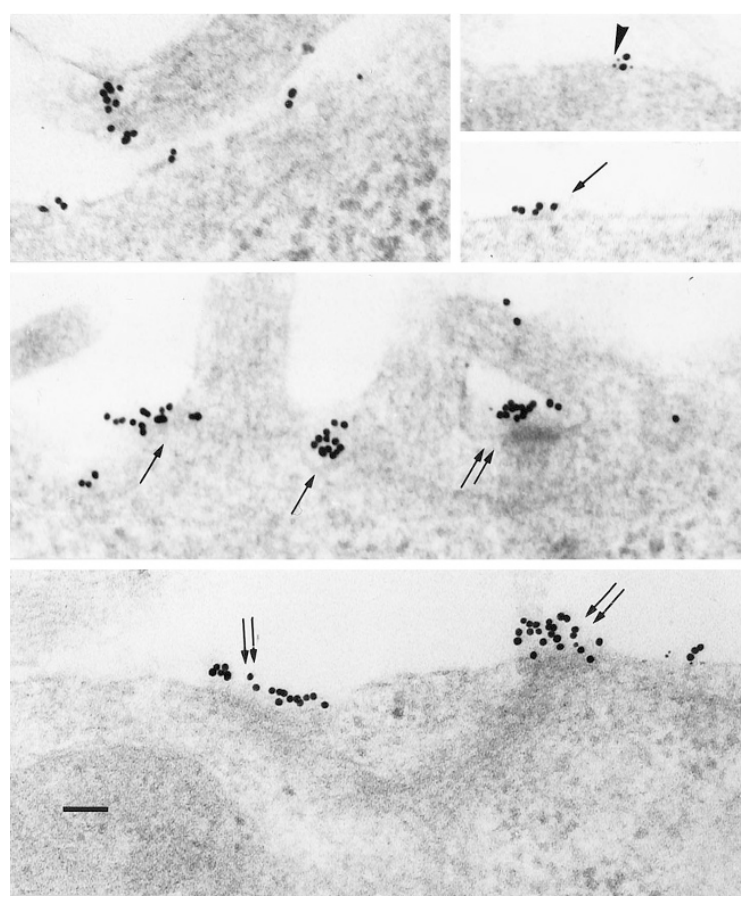

Figure 4.

Time-lapsed, double-stained immunoelectron micrographs of DJM-1 cells treated with a pulse of PV-lgG and 5-nm-gold staining and chased for a 60-minute period, followed by a second pulse of 5-minute staining with PV-IgG and 10-nm gold particles. Most of clusters of gold particles on the nondesmosomal cell surface were composed of 10-nm-sized particles, suggesting that Dsg3 molecules were newly expressed on the cell surface during the chase period of 60 minutes. These clusters appeared in the form of simple clusters without KIF-attachment (arrows) and half-desmosome-like clusters with KIF attachment (double arrows). Some clusters were also labeled with 5-nm gold particles (arrowhead). Scale bar $=0.5 \mu \mathrm{m}$.

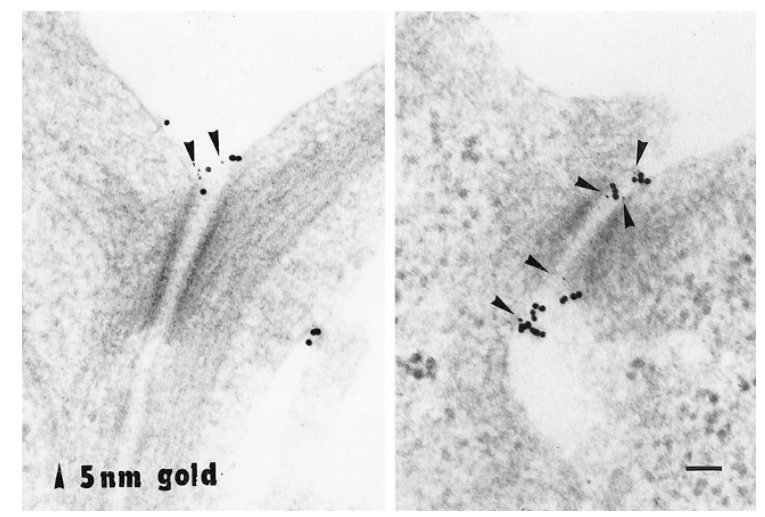

\section{Figure 5.}

Time-lapsed, double-stained immunoelectron micrographs of cells treated with a pulse of PV-lgG and 5-nm-gold staining and chased for a 60 -minute period, followed by a pulse of 5-minute staining with PV-IgG and $10-\mathrm{nm}$ gold particles. Desmosomes were labeled with both $5-\mathrm{nm}$ and $10-\mathrm{nm}$ gold particles, indicating that they had been continuously expressed on the cell surface during the chase period of 60 minutes. Scale bar $=0.5 \mu \mathrm{m}$.

nogold staining (Jones et al, 1986) revealed that PV-IgG binds throughout the plasma membrane of keratinocytes including desmosomes. More recent pre- and post-embedding immunogold methods have revealed extensive localization of Dsg3 on the desmosomal, but not on the nondesmosomal, plasma mem- brane surfaces, suggesting that Dsg3 localizes exclusively to desmosomes (Akiyama et al, 1991; Iwatsuki et al, 1989; Karpati et al, 1993; Rappersberger et al, 1992; Zhou et al, 1997). The present study by immunoelectron microscopy illustrated that Dsg3 was distributed on the nondesmosomal keratinocyte surfaces in two small cluster types, ie, simple clusters without KIF-attachment and half-desmosome-like clusters with KIF-attachment on the intracellular surface of the plasma membrane. Serial sections confirmed that these structures are not peripheral parts of desmosomes.

To more precisely localize Dsg3 temporally within the cell during the formation of the desmosome, we used a novel time-lapsed double immunostaining technique to determine the age and fate of Dsg3 on the cell surface. This technique comprises a combination of 5-nm gold to label "early" Dsg3-containing complexes and 10-nm gold to label Dsg3 produced "recently" or "later"; ie, following a 60-minute chase. This pulse immunogold technique revealed that after the 60-minute chase, nearly all of these clusters of labels on the cell surface were composed of 10-nm gold, suggesting that these Dsg3 clusters were newly generated and not from split desmosomes or their breakdown products. These results suggested that newly synthesized Dsg3 is distributed early on the nondesmosomal cell surface. Besides, 5-nm goldlabeled clusters in the inner surface of the endosomal membrane were found, suggesting that Dsg3-PV-IgG immune complexes are internalized as endosomes within 60 minutes after PV-lgG was exposed. We confirmed also, by time-lapsed double labeling immunofluorescence microscopy, that Dsg3 is freely floating on the nondesmosomal cell surface and that PV-IgG causes its lateral aggregation and internalization into the cell within 60 minutes after PV-IgG binding to the cell surface components.

Previous electron microscopy studies have shown that when keratinocytes are either transferred to a culture medium depleted of $\mathrm{Ca}^{++}$or treated with trypsin, split desmosomes (or half-desmosomes) are produced, and rapidly internalize as endosomes associated with plaque-attached KIF bundles, and a punctuated pattern of desmosome-derived vesicles (endosomes) are consistently observed in antidesmoplakin antibody-stained cells by immunofluorescence microscopy (Duden and Franke, 1988; Franke et al, 1982; Kartenbeck et al, 1982, 1991; Overton, 1968). The internalized, split-desmosome-associated endosomes are suggested to be detached from the plaque soon after internalization, with retraction of KIF to the juxtanuclear regions (Kartenbeck et al, 1982). Internalization of half-desmosomes, referred to as desmosome-associated vacuoles (DSV), also was seen in Madin-Darby canine kidney (MDCK) cells. The DSV are presumed to be involved in the degradation, rather than the assembly, of desmosomes as one of the general mechanisms for removing desmosomes during normal physiologic remodeling of tissue architecture (Burdett, 1993). 
Similar to these responses seen in MDCK cells (Burdett, 1993), or when keratinocytes are transferred into $\mathrm{Ca}^{++}$-free culture medium (Kartenbeck et al, 1982, 1991) or treated with trypsin (Overton, 1968), it has been shown in vivo that desmosomes begin to split into half-desmosomes leading to their internalization at 18 to 24 hours after passive transfer of PV-IgG to neonatal mice (Takahashi et al, 1985). Similar observations were reported with immunogold labeling of the lesional skin of PV patients (Iwatsuki et al, 1989) and in neonatal mice injected with PV-IgG (Karpati et al, 1993). Injection of PF-IgG also causes internalization of half-desmosomes in granular cells of some neonatal mice after 24 hours (Futamura et al, 1989). Because such internalization of half-desmosomes was not observed until at least 18 to 24 hours after binding of pemphigus antibodies, this event may represent a later response of keratinocyte to PV- or PF-IgG stimulation. Furthermore, because the internalized endosomes bearing half-desmosomes that are seen in $\mathrm{Ca}^{++}$switch to low trypsin treatment, or in MDCK cells are morphologically similar to those seen in PV-IgG or PF-IgG treatment, it appears feasible that these responses to PV- or PF-IgG may be a result of, but not cause for, desmosomal splitting. It is also feasible that the activation of plasminogen activator and plasmin induced by PV-lgG binding (Hashimoto et al 1983; Morioka et al, 1981; Seishima et al, 1997) may be involved in desmosomal splitting and lead to internalization of half-desmosomes, even though this may not be a primary causative event in the blistering of pemphigus (Kitajima et al, 1999; Mahoney et al, 1999).

The present study shows that since all desmosomes are simultaneously labeled with both "early" 5-nm gold and "late" 10-nm gold, whereas all internalized gold-label clusters were simple Dsg3 clusters without KIF, rather than half-desmosome-like structures, desmosomes are not split for at least 60 minutes following incubation with PV-lgG. Furthermore, since the internalization of half-desmosomes was not detected at this time, even though simple clusters of Dsg3 were internalized, within 60 minutes after PV-IgG stimulation, the internalized half-desmosomes, which have been found in the biopsied specimens from patients with PV (Iwatsuki et al, 1989) or experimental PV-mice (Takahashi et al, 1985), are not comparable to those internalized structures observed in the present study. Moreover, the internalization of half-desmosomes may not be the primary effect of PV-IgG stimulation leading to cell-cell detachment, but rather may reflect a secondary or final stage of cell-cell detachmentassociated events.

Prior works also have shown that half-desmosomelike structures that are not produced by splitting desmosomes are formed in cultured $\mathrm{HaCaT}$ cells and A-431 carcinoma cells. In HaCaT cells, Demlehner et al (1995) have shown that half-desmosomes, which contain Dsgs and the plaque proteins, are formed in low $\mathrm{Ca}^{++}$culture medium and also in normal $\mathrm{Ca}^{++}$ grown cells when they are uncoupled. They also showed that if these half-desmosomes are not coupled with each other to form desmosomes in low
$\mathrm{Ca}^{++}$medium, they are taken up endocytotically, a process referred to as a coordinated Sisyphus cycle. From these observations, they suggested that, in the absence of intercellular contacts, assembly of desmosomal proteins at the cell surface takes place, resulting in transient half-desmosomes, and proposed that these half-desmosomal structures are the general cell structure of epithelial cells (Demlehner et al, 1995). Besides this, the existence of cell-surface exposed Dsg2 has also been clearly shown in the absence of desmosomes in uncoupled $\mathrm{HaCaT}$ cells by immunofluorescence and immunoelectron microscopy using antibodies to the extracellular epitopes of Dsg2, suggesting that nondesmosome-bound Dsg2 may be dispersed and clustered in half-desmosomes on the cell surface (Schaefer et al, 1996). A-431 cells, when passed through low $\mathrm{Ca}^{++}$medium in the absence of stable junctions, are able to assemble halfdesmosome-like structures on the plasma membrane, suggesting that they are not products of splitting desmosomes (Duden and Franke, 1988). Our present study confirmed these findings by time-lapsed double-stained immunoelectron microscopy in DJM-1 cells. In the DJM-1 cells used in the present study, half-desmosome-like structures were evident in normal $\mathrm{Ca}^{++}$-containing culture medium without any treatments to induce desmosome splitting. Therefore, it appears reasonable to speculate that Dsg3 is first assembled into small clusters without KIF, followed by KIF-attachment, and finally full desmosomal assembly. Furthermore, PV-IgG causes the internalization of Dsg3 at an early stage of simple Dsg3 cluster formation before KIF-attachment, because even when cells were treated with PV-lgG, no internalization of halfdesmosome-like structures was seen within 60 to 180 minutes.

Previously, we traced the fate of Dsg3 and other desmosomal molecules after PV-IgG binding to the Dsg3 on the cell surface in DJM-1 cells (Aoyama et al, 1999; Aoyama and Kitajima, 1999). After cells were incubated with PV-IgG for different periods of time, cells were fractionated into Triton $X-100$ soluble (membrane) and Triton X-100 insoluble (cytoskeleton) fractions. Immunoblotting and immunofluorescence microscopy using antibodies against Dsg1, Dsg3, plakoglobin (PG), desmoplakin 1 (Dpk1), and cytokeratins revealed that Dsg3 was markedly depleted from the membrane fraction within 20 minutes after PV-lgG treatment, whereas no reduction of Dsg3 was detected in the cytoskeleton fraction. However, a $30-$ hour incubation with PV-lgG caused a marked disappearance of Dsg3, but not other desmosomal molecules, from cytoskeleton fractions. Furthermore, double-staining immunofluorescence microscopy revealed that Dsg3 was particularly depleted from the desmosomes, which were still maintained 30 hours after PV-IgG treatment, whereas the desmosomal contacts of Dsg1, Dpk1, PG, and keratin filaments were unaffected. The present study provides evidence for an early keratinocyte response to PV-IgG, ie, a rapid internalization of Dsg3-PV-IgG immune complexes as endosomes, which could help to account for 
the formation of Dsg3-deleted desmosomes in PV patient epidermis.

In summary, the results of this study showed that Dsg3 was initially expressed on the nondesmosomal cell surface as small clusters, followed by attachment with KIF, and confirmed previous observations that half-desmosome structures were first formed and subsequently assembled into desmosomes on the cell surface (Demlehner et al, 1995). Furthermore, we demonstrated that PV-IgG caused lateral aggregations, detectable as dots by immunofluorescence, and internalization of these Dsg3 clusters, detectable by immunoelectron microscopy, suggesting that these responses may impair the assembly of Dsg3 to desmosomes, resulting in the formation of Dsg3-deleted desmosomal complexes.

\section{Materials and Methods}

\section{Antibodies and Pemphigus IgG}

Sera from 4 patients with PV and from healthy volunteers without any skin disease were used for the current experiments. All sera from patients with PV were confirmed to react only with Dsg3, and not with Dsg1, by antigen-specific enzyme-linked immunosorbent assay using baculovirus-expressed recombinant peptides of extracellular domains of Dsg3 and Dsg1 (Ishii et al, 1997). This was also confirmed by immunoblotting using total cell extracts of cultured DJM-1 cells and normal human keratinocytes (Aoyama and Kitajima, 1999). All sera were heated to $56^{\circ} \mathrm{C}$ for 30 minutes to inactivate complement, and stored at $-80^{\circ} \mathrm{C}$. IgG fractions were isolated from these sera by a Mab Trap Gll kit (Pharmacia AB, Uppsala, Sweden). The purity of isolated IgG was checked by immunoelectrophoresis. Thus, purified IgG (PV-IgG) from PV sera was used for the stimulation of the cultured keratinocytes by adding it to culture medium, and PV-IgG obtained from serum of a PV patient with the highest titer was used for the detection of Dsg3.

Fluorophore-conjugated, affinity-purified secondary antibodies against human IgG were purchased from ICN Pharmaceuticals Inc. (Costa Mesa, California). Colloidal gold (5-nm and 10-nm) conjugates consisting of affinity-purified IgG antibodies against human IgG were obtained from Nanoprobes Inc. (Yaphank, New York).

\section{Cell Cultures}

An isolated cell line (DJM-1) from human skin squamous cell carcinoma was used in the present study (Kitajima et al, 1988, 1992). DJM-1 cells were cultured in MEM (Life Technologies, Inc., Rockville, Maryland) containing $10 \% \mathrm{FCS}, 0.4 \mathrm{mg} / \mathrm{ml}$ hydrocortisone, 10 $\mathrm{ng} / \mathrm{ml}$ epidermal growth factor, $84 \mathrm{ng} / \mathrm{ml}$ cholera toxin, $100 \mathrm{mg} / \mathrm{ml}$ kanamycin, and $1.8 \mathrm{~mm} \mathrm{Ca}^{++}$. At $80 \%$ to $90 \%$ confluence, cells were exposed to PBS containing $0.25 \%$ trypsin and $10 \mathrm{~mm}$ EDTA for 10 minutes at $37^{\circ} \mathrm{C}$. They were resuspended in MEM with 10\% FCS or HuMedia-KG2, cultured for 2 days, and used as described below.

\section{Immunofluorescence Microscopy}

Short Pulse Staining with Anti-Dsg3 Antibodies. The cells, cultured on the glass coverslips, were subjected to pulse incubation in culture medium containing PV-IgG (ie, anti-Dsg3) for 3 minutes and rinsed with culture medium three times, followed by incubation in fresh culture medium containing antihuman IgG conjugated with FITC for 5 minutes. Immediately after this treatment, cells were rinsed with PBS at $37^{\circ} \mathrm{C}$ and fixed by dipping for 7 minutes in $3 \%$ paraformaldehyde in $0.1 \mathrm{~m}$ sodium phosphate buffer.

To chase the fate or redistribution of Dsg3 after binding with PV-lgG, these pulse-PV-IgG-treated and pulse-FITC-stained cells were incubated in antibodyfree culture medium for an additional 60 minutes and fixed with $3 \%$ paraformaldehyde in $0.1 \mathrm{~m}$ sodium phosphate buffer. As an alternative experiment, after incubation in antibody-free medium for 60 minutes, pulsePV-IgG-treated cells were fixed with $3 \%$ paraformaldehyde in $0.1 \mathrm{M}$ sodium phosphate buffer for 7 minutes, followed by treatment with $0.05 \%$ Triton X-100, which leads a second antibody to penetrate into the intracellular spaces and endosomes. Thus, fixed cells were stained with antihuman IgG-FITC (45 min). Negative controls were carried out at the same time using normal human IgG.

Time-Lapsed Double Staining of "Early Dsg3" and "Late Dsg3". In order to determine the distribution of "early" Dsg3, which had been expressed on the cell surface before starting the 3-minute-pulse treatment, and the "late" or "new" Dsg3 expressed during the 60-minute chase, cells were first pulse-treated with PV-IgG (3 min), stained with antihuman IgG-FITC in culture medium (5 min), and incubated for 60 minutes in antibody-free culture medium. Then, these cells were briefly reincubated with PV-lgG (5 min) after the 60-minute chase in antibody-free culture medium, and stained with fresh medium containing antihuman IgGRhodamine (Rod) goat antibodies (5 min). Because the second staining with PV-lgG (anti-Dsg3) and antihuman IgG-Rod was carried out in living cells in culture medium, if "early Dsg3 molecules" were internalized as endosomes, the anti-Dsg3 and antihuman IgG Rod could not reach the internalized Dsg3 molecules. Therefore, spots stained only with FITC indicate the internalized antigens, and those stained only with Rod indicate "late" Dsg3 expressed during the 60-minute chase.

\section{Immunoelectron Microscopy}

Natural Distribution of Dsg3 on the Cell Surface. The cells cultured on the glass coverslips were rinsed with PBS at $37^{\circ} \mathrm{C}$ and fixed by dipping for 7 minutes in $3 \%$ paraformaldehyde in $0.1 \mathrm{M}$ sodium phosphate buffer. After washing thoroughly with PBS, cells were incubated with $1 \mathrm{mg} / \mathrm{ml}$ of PV-lgG (ie, anti-Dsg3) or normal human IgG (as controls) for 12 hours and stained with antihuman goat IgG conjugated with 10-nm gold particles after washing with PBS and PBS containing 1\% goat albumin. Then, cells were rinsed with PBS at 
$37^{\circ} \mathrm{C}$ and fixed by dipping for 7 minutes in $3 \%$ paraformaldehyde in $0.1 \mathrm{M}$ sodium phosphate buffer. This prefixed cell labeling illustrated the natural physiological distribution of Dsg3 molecules on the cell surface of DJM-1 cells and normal human keratinocytes.

Pulse-Stimulation with PV-IgG and the Chase of $P V-I g G-B o u n d D s g 3$. Cells were pulse-stimulated with PV-IgG or normal human IgG for 3 minutes, washed with fresh culture medium, and labeled with 5-nm gold conjugated with antihuman IgG in culture medium for 5 minutes, followed by immediate washing with fresh culture medium. Thus, pulse-labeled cells were chased in antibody-free fresh culture medium for 15 or 60 minutes and fixed with 3\% paraformaldehyde in $0.1 \mathrm{~m}$ sodium phosphate buffer at $37^{\circ} \mathrm{C}$ for 7 minutes.

Time-Lapsed Double Staining of "Early Dsg3" and "Late Dsg3". To determine ultrastructurally the fate of Dsg3 bound by PV-IgG and the distribution of the "late" Dsg3 molecules expressed on the cell surface during a rather short period (60 min), time-lapsed double stainings of "early" Dsg3 and the "late" Dsg3 molecules were carried out. First, cells were pulse incubated in culture medium containing PV-IgG for 3 minutes and stained with antihuman IgG labeled with 5-nm gold particles for 5 minutes after washing with fresh culture medium. Then, after washing thoroughly with fresh culture medium, these cells were further incubated in antibody-free culture medium for 60 minutes. After a 60-minute chase incubation, 3-minute pulse-labeled cells were briefly reincubated with PVIgG (5 min) and stained with antihuman IgG-10-nm gold antibodies for 5 minutes before fixation or for 12 hours after fixation (7 min) with $3 \%$ paraformaldehyde in $0.1 \mathrm{M}$ sodium phosphate buffer. This method indicated that $10-\mathrm{nm}$ gold particles, when concurrent labels with 5-nm gold were lacking, represent "late" Dsg3 clusters newly expressed on the cell surface that had arisen during the 60-minute chase period. It also indicated that the 5-nm gold label contrived to represent older clusters of Dsg3 molecules formed before the chase period. Negative controls were carried out at the same time using normal human IgG.

Methods for Observation by Electron Microscopy. All samples were fixed with 3\% paraformaldehyde in $0.1 \mathrm{M}$ sodium phosphate buffer, $\mathrm{pH} 7.4$, for 5 to 7 minutes at $37^{\circ} \mathrm{C}$, followed by fixation with a $3 \%$ aqueous solution of osmium tetroxide $(\mathrm{OsO} 4)$ for 4 hours at $37^{\circ} \mathrm{C}$. Fixed samples were embedded in Epon 812 (Nisshin EM Company, Ltd., Tokyo, Japan). Ultrathin sections were made parallel to the bottom of the dish and counterstained with uranyl acetate and lead citrate. For some of specimens, 4 to 5 serial thin sections (100 $\mathrm{nm}$ thick) were made.

\section{References}

Akiyama M, Hashimoto T, Sugiura M, and Nishikawa T (1991). Ultrastructural localization of pemphigus vulgaris and pemphigus foliaceus antigens in cultured human squamous carcinoma cells. Br J Dermatol 125:233-237.
Amagai M, Koch PJ, Nishikawa T, and Stanley JR (1996). Pemphigus vulgaris antigen (Desmoglein 3 ) is localized in the lower epidermis, the site of blister formation in patients. $\mathrm{J}$ Invest Dermatol 106:351-355.

Amagai M (1995). Adhesion molecules. I: Keratinocytekeratinocytes interaction; cadherins and pemphigus. J Invest Dermatol 104:146-152.

Aoyama $Y$ and Kitajima $Y$ (1999). Pemphigus vulgaris-lgG causes a rapid depletion of desmoglein 3 (Dsg3) from the triton X-100 soluble pools, leading to the formation of Dsg3depleted desmosomes in a human squamous carcinoma cell line, DJM-1 cells. J Invest Dermatol 112:67-71.

Aoyama Y, Owada MK, and Kitajima Y (1999). A pathogenic autoantibody, pemphigus lgG, induces phosphorylation of desmoglein3, and its dissociation from plakoglobin in cultured keratinocytes. Eur J Immunol 29:2233-2240.

Arnemann J, Sullivan KH, Magee AI, King IA, and Buxton RS (1993). Stratification related expression of isoforms of the desmosomal cadherins in human epidermis. J Cell Sci 104: $741-750$.

Bedane C, Prost C, Thomine E, Intrator L, Joly P, Caux F, Blecker $M$, Bernard $P$, Leboutet $M J$, Tron $F$, Lauret $P$, Bonnetblanc JM, and Dubertret L (1996). Binding of autoantibodies is not restricted to desmosomes in pemphigus vulgaris: Comparison of 14 cases of pemphigus vulgaris and 10 cases of pemphigus foliaceus studied by Western immunoblot and immunoelectron microscopy. Arch Dermatol Res 288:343-352.

Burdett ID (1993). Internalization of desmosomes and their entry into the endocytic pathway via late endosomes in MDCK cells. Possible mechanisms for the modulation of cell adhesion by desmosomes during development. J Cell Sci 106:1115-1130.

Buxton RS, Cowin P, Franke WW, Green KJ, King LA, Koch PJ, Magree Al, Rees DA, Stanley R, and Steinberg MS (1993). Nomenclature of desmosomal cadherins. J Cell Biol 121: 481-483.

Cowin P, Kapprell HP, Franke WW, Tamkun J, and Hynes RO (1986). Plakoglobin: A protein common to different kinds of intercellular adhering junctions. Cell 46:1063-1073.

Demlehner MP, Shaefer S, Grund C, and Franke WW (1995). Continual assembly of half-desmosomal structures in the absence of cell contacts and their frustrated endocytosis: A coordinated Sisyphus cycle. J Cell Biol 131:745-760.

Duden R and Franke WW (1988). Organization of desmosomal plaque proteins in cells growing at low calcium concentrations. J Cell Biol 107:1049-1063.

Franke WW, Moll R, Shiller DL, Schmid E, Kartenbeck J, and Mueller H (1982). Desmoplakins of epithelial and myocardial desmosomes are immunologically and biochemically related. Differentiation 23:226-237.

Futamura S, Martins C, Rivitti EA, Labib RS, Diaz LA, and Anhalt GJ (1989). Ultrastructural studies of acantholysis induced in vivo by passive transfer of IgG from endemic pemphigus foliaceus (Fogo Selvagem). J Invest Dermatol 93:480-485.

Hashimoto K, Shafran KM, Webber PA, Lazarus GS, and Singer KH (1983). Anti-cell surface pemphigus antibody stimulates plasminogen activator activity of human epidermal cells. A mechanism for the loss of epidermal cohesion and blister formation. J Exp Med 157:259-272. 
Heid HW, Schmidt A, Zimbelmann R, Schafer S, WinterSimanowski S, Stumpp S, Keith M, Figge U, Schnolzer M, and Franke WW (1994). Cell type-specific desmosomal plaque proteins of the plakoglobin family: Plakophilin 1 (band 6 protein). Differentiation 58:113-131.

Hieda Y, Tsukita S, and Tsukita S (1989). A new high molecular mass protein showing unique localization in desmosomal plaque. J Cell Biol 109:1511-1518.

Ishii K, Amagai M, Hall PP, Hashimoto T, Takayanagi A, Gamou S, Shimizu N, and Nishikawa T (1977). Characterization of autoantibodies in pemphigus using antigen-specific enzymelinked immunosorbent assays with baculovirus-expressed recombinant desmogleins. J Immunol 159:2010-2017.

Iwatsuki K, Takigawa M, Imaizumi S, and Yamada M (1989). In vivo binding site of pemphigus vulgaris antibodies and their fate during acantholysis. J Am Acad Dermatol 20:578-582.

Jones JC, Yokoo KM, and Goldman RD (1986). A cell surface desmosome-associated component: Identification of a tissue-specific cell adhesion molecule. Proc Natl Acad Sci USA 83:7282-7286.

Karpati S, Amagai M, Prussick R, Cehrs K, and Stanley JR (1993). Pemphigus vulgaris antigen, a desmoglein type of cadherin, is localized within keratinocyte desmosomes. J Cell Biol 122:409-415.

Kartenbeck J, Schmelz M, Franke WW, and Geiger B (1991). Endocytosis of junctional cadherins in bovine kidney epithelial (MDBK) cells cultured in low $\mathrm{Ca}^{2+}$ ion medium. J Cell Biol 113:881-892.

Kartenbeck J, Schmid E, Franke WW, and Geiger B (1982). Different modes of internalization of proteins associated with adherens junctions and desmosomes: Experimental separation of lateral contacts induces endocytosis of desmosomal plaque material. EMBO J 1:725-732.

Kitajima $Y$ (1996). Adhesion molecules in the pathophysiology of bullous diseases. Eur J Dermatol 6:399-405.

Kitajima Y, Aoyma Y, and Seishima M (1999). Transmembrane signaling for adhesive regulation of desmosomes and hemidesmosomes, and for cell-cell detachment induced by pemphigus IgG in cultured keratinocytes: Involvement of protein kinase C. J Investig Dermatol Symp Proc 4:137-144.

Kitajima Y, Inoue S, and Yaoita H (1987). Effects of pemphigus antibody on the regeneration of cell-cell contact in keratinocyte culture grown in low to normal $\mathrm{Ca}^{++}$concentration. J Invest Dermatol 89:167-171.

Kitajima $\mathrm{Y}$, Inoue S, Nagao S, Nagata K, Yaoita H, and Nozawa $Y$ (1988). Biphasic effects of 12-0tetradecanoylphorbol-13-acetate on the cell morphology of low calcium-grown human epidermal carcinoma cells: Involvement of translocation and down regulation of protein kinase C. Cancer Res 48:964-970.

Kitajima Y, Owaribe K, Nishizawa Y, Jokura Y, and Yaoita $\mathrm{H}$ (1992). Phorbol ester-and calcium-induced reorganization of $180-\mathrm{kDa}$ bullous pemphigoid antigen on the ventral surface of cultured human keratinocytes as studied by immunofluorescence and immunoelectron microscopy. Exp Cell Res 203:17-24.

Koch PJ and Franke WW (1994). Desmosomal cadherins: Another growing multigene family of adhesion molecules. Curr Opin Cell Biol 6:682-687.

Koch PJ, Mahoney MG, Ishikawa H, Pulkkinen L, Uitto J, Shultz L, Murphy GF, Whitaker-Menezes D, and Stanley JR
(1997). Targeted disruption of the pemphigus vulgaris antigen (desmoglein 3) gene in mice causes loss of keratinocyte cell adhesion with a phenotype similar to pemphigus vulgaris. J Cell Biol 137:1091-1102.

Legan PK, Collins JE, and Garrod DR (1992). The molecular biology of desmosomes and hemidesmosomes, "What's in a name?" Bioessays 14:385-393.

Mahoney MG, Wang ZH, and Stanley JR (1999). Pemphigus vulgaris and pemphigus foliaceus antibodies are pathogenic in plasminogen activator knockout mice. J Invest Dermatol 113:22-25.

Morioka S, Naito K, and Ogawa $\mathrm{H}$ (1981). The pathogenic role of pemphigus antibodies and proteinase in epidermal acantholysis. J Invest Dermatol 76:337-341.

Mueller H and Franke WW (1983). Biochemical and immunological characterization of desmoplakins I and II, the major polypeptides of the desmosomal plaque. J Mol Biol 163:647671.

Ouyang P and Sugrue SP (1996). Characterization of pinin, a novel protein associated with the desmosome-intermediate filament complex. J Cell Biol 135:1027-1042.

Overton $J$ (1968). The fate of desmosomes in trypsinized tissue. J Exp Zool 168:203-214.

Patel HP, Diaz LA, Anhalt GJ, Labib RS, and Takahashi Y (1984). Demonstration of pemphigus antibodies on the cell surface of murine epidermal cell monolayers and their internalization. J Invest Dermatol 83:409-415.

Rappersberger K, Roos N, and Stanley JR (1992). Immunomorphologic and biochemical identification of the pemphigus foliaceus autoantigen within desmosomes. J Invest Dermatol 99:323-330.

Ruhrberg C, Hajibagheri MA, Parry DA, and Watt FM. (1997). Periplakin, a novel component of cornified envelopes and desmosomes that belongs to the family and forms complexes with envoplakin. J Cell Biol 139:1835-1849.

Ruhrberg C, Hajibagheri MA, Simon M, Dooley TP, and Watt FM (1996). Envoplakin, a novel precursor of the cornified envelope that has homology to desmoplakin. J Cell Biol 134:715-729.

Ruhrberg C and Watt FM. (1997). The plakin family: versatile organizers of cytoskeletal architecture. Curr Opin Genet Dev 7:392-397.

Seishima M, Satoh S, Nojiri M, Osada K, and Kitajima Y (1997). Pemphigus IgG induces expression of urokinase plasminogen activator receptor on the cell surface of cultured keratinocytes. J Invest Dermatol 109:650-655.

Schaefer S, Stumpp S, and Franke WW (1996). Immunological identification and characterization of the desmosomal cadherin Dsg2 in coupled and uncoupled epithelial cells and in human tissues. Differentiation 60:99-108.

Shimizu H, Masunaga T, Ishiko A, Kikuchi A, Hashimoto T, and Nishikawa T (1995). Pemphigus vulgaris and pemphigus foliaceus sera show an inversely graded binding pattern to extracellular regions of desmosomes in different layers of human epidermis. J Invest Dermatol 105:153-159.

Stanley JR (1992). Cell adhesion molecules as targets of autoantibodies in pemphigus and pemphigoid bullous diseases due to defective epidermal cell adhesion. Adv Immunol 53:291-325. 
Takahashi Y, Patel HP, Labib RS, Diaz LA, and Anhalt GJ (1985). Experimentally induced pemphigus vulgaris in neonatal BALB/c mice: A time-course study of clinical, immunologic, ultrastructural, and cytochemical changes. J Invest Dermatol 84:41-46.

Takigawa M, Imamura S, and Ofuji S (1978). Surface distribution of pemphigus antibody-binding substance(s) on isolated guinea pig epidermal cells. An immunoferritin electron microscopic study. J Invest Dermatol 71:182-185.

Tsukita S and Tsukita S (1985). Desmocalmin: A calmodulinbinding high molecular weight protein isolated from desmosomes. J Cell Biol 101:2070-2080.

Wiche G, Becker B, Luber K, Weitzer G, Castanon MJ, Hauptmann R, Stratowa C, and Stewart M (1991). Cloning and sequence of rat plectin indicates a $466-\mathrm{kD}$ polypeptide chain with a three-domain structure based on a central alpha-helical coiled coil. J Cell Biol 114:83-99.
Wolff K and Schreiner E (1971). Ultrastructural localization of pemphigus autoantibodies within the epidermis. Nature 229: $59-61$.

Zhou S, Ferguson DJP, Allen J, and Wojnarowska F (1997). The location of binding sites of pemphigus vulgaris and pemphigus foliaceus autoantibodies: A post-embedding immunoelectron microscopic study. Br J Dermatol 136:878883. 